\title{
QC 小组活动在计划阶段的失误分析与应对
}

\section{Error Analysis and Response of QC Group Activities in Planning Stage}

\author{
熊成华 1,2 \\ Chenghua Xiong ${ }^{1,2}$ \\ 1.福建省松溪县中等职业技术学校 中国・福建 南平 $353500 ； 2$. 福建省松溪县环城建筑公司 中国・福建 南平 353500 \\ 1. Fujian Songxi Secondary Vocational and Technical School, Nanping, Fujian, 353500, China; \\ 2. Fujian Songxi Construction Company, Nanping, Fujian, 353500, China
}

摘 要: QC 小组在越来越多的企业管理中应用, 其运作正确与否很大程度上影响了质量管理效益, 因此, 减少运作环节中的 失误成了一个很重要的课题。QC 小组工作过程是 PDCA(计划、执行、检查、行动)的四阶段工作过程, 而 P(计划)过程在全过 程中起基础性的前提作用，论文用意在于对(问题型)QC 小组在 P 阶段运作环节中碰到的常见问题进行分析梳理, 并给出相 应处理对策, 总结各环节运作要点, 以应对工作失误,提高 QC 小组工作效能。

\begin{abstract}
Nowadays, more and more QC group has been applied in enterprise management. It affects the efficiency of enterprise managing quality. Therefore, reducing the mistakes in the operation has become a very important subject. The working process of the QC group is a four-stage working process of the PDCA cycle (Plan, Do, Check and Action). Meanwhile, the P (plan) stage plays a fundamental role in the whole group. The main purpose of this paper is to analyze and sort out the common problems encountered by the QC group in the operation of the P stage, and to summarize the main points of each link in order to deal with the mistakes and improve the efficiency of the QC group work.
\end{abstract}

关键词: $\mathrm{QC}$ 小组; 失误分析与应对; P 过程

Keywords : QC group; error analysis and response; P process

\section{DOI : 10.36012/etr.v2i8.2520}

\section{$1 \mathrm{QC}$ 小组}

QC 小组,也称质量管理小组,按中国质量管理小组活动 准则定义, 是指由生产、服务及管理等工作岗位的员工自愿 结合组成几个人一圈的小团体, 他们围绕着组织的经营战 略、方针目标和现场存在的问题进行活动, 目的在于改进质 量、降低消耗、改善环境、提高人的素质和经济效益, 运用的 工具是质量管理理论和方法 ${ }^{[1]}$ 。小组全体合作、集思广益解决 工作管理、现场、文化等方面发生的问题(课题)。它面对的问 题 (课题) 类型是问题解决型与创新型两种。

\section{2 问题解决型课题和对应 QC 小组运作程序}

问题解决型课题, 是指针对已经发生不合格或不满意的 生产、服务或管理现场存在的问题进行质量改进, 从而选择 的质量管理小组课题。其活动程序根据目标来源不同, 人们 把它分成两类程序: 自定目标值类和指令性目标类 ${ }^{[2]}$ 。前者程
序步骤是:(1)选择课题(2)现况调查(3)设定目标(4)分析原因(5) 确定主要原因(6)制定对策(7)对策实施(8)效果检查(9)达到目 标(若否返回原因分析)(10制定巩固措施并总结和作下一步打 算;后者程序步骤是: (1)选择课题(2)设定目标(3)目标可行性 分析(4)原因分析(5)确定主要原因(6)制定对策(7)对策实施(8) 效果检查(9)达到目标(若否返回原因分析)(10制定巩固措施并 总结和作下一步打算。

这些程序过程人们又喜欢把它分成 $P 、 D 、 C 、 A$ 四个过 程。 $\mathrm{P}$ (计划)过程是“(1)选择课题……6制定对策”; $\mathrm{D}($ 执 行)过程是“(7)对策实施”; $\mathrm{C}$ (检查)过程是“(8)效果检查 $\rightarrow(9)$

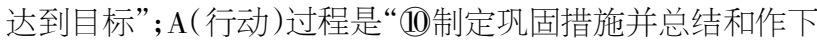
一步打算”。其中 $\mathrm{P}$ 工作过程是四个过程中的基础过程,处理 得当与否对 $Q C$ 小组的后序工作成效有着重要的作用 ${ }^{[3]}$ 。本 文仅就 P 工作过程中常见问题进行分析与思考, 提出应对措 施办法,与同行分享。 


\section{$3 Q C$ 小组活动在 $P$ 过程的常见问题及应对}

\section{1 课题选择方面}

(1)这一环节工作在于: 针对存在的问题和改进对象, 结 合实际, 选择适宜课题。

(2)常见失误: a. 课题名称空泛,如“改善工程环境,推行 绿色施工”; b. 课题名称不明确, 如 “使用 PDCA 循环, 解决工 程难题”; c. 使用 “对策+效果”等词句作课题名称, 如“优化工 程中施工配比, 确保混凝土再次利用质量”; d. 课题内容过 多, 如名称“大面积胶合板合理使用、模板支撑科学设计及质 量的控制”, 其中就涵盖 3 个部分内容; e. 课题名称缺少可比 性,如“保证楼梯踏步的施工质量”; f. 选题理由不充分。

(3)失误分析与应对: 按准则要求所选课题, 既要是小组 活动才能解决的, 又要是小组力所能及的。选题的原则根据 “实、小、活、新”,以“实”为主, 课题一般宜“小”不宜“大”; 选 定的课题名称要明确、简洁, 对正要解决的问题且不可以抽 象,且课题的名称应该一目了然;禁止采用“手段+目的”和 “口号式”; 选题理由的陈述只需简要地表述上级(或标准)要 求指标, 自己部门目前实际实现多少, 用简要数据明确表达 出差距, 直接陈述选择这一课题的必要性和目的, 意思表达 就充分到位了。

(4)选题要点是: 名称要直接、表达特征值; 在能力范围 内, 宜小不宜大; 理由明确、简洁。

(5)选题采取的工具有: 分层法、排列图、调查表、头脑风 暴法、简易图表等。

\section{2 现况调查方面}

(1)这一环节工作在于:掌握问题严重到什么程度,对收集 到的数据运用整理、分析的方法进行梳理, 直至找出症结来。

(2)常见失误: a. 把调查对象当作原因; b. 直接分析课题; c. 获取、整理、分析调查数据不当; $\mathrm{d}$. 统计工具、方法不适用。

(3)失误分析与应对: 现况调查的目的是要确定实际情况 对课题的影响程度, 并为确定目标提供现实依据, 需要调查 的是“现象”, 而不是“原因”; 没有对课题做现况调查, 就直接 进行分析, 或根据既往经验和体会, 且在无现况调查资料支 持下, 凭空主观想象、判断下结论,这些都不符合 QC 质量准 则的求真、务实要求; 数据应是在小组开始活动的时间节点 上最接近的现实反映, 并且应剔除不关联数据; 现况调查必 须以真实、正确、准确和有效为原则, 择选合适的统计方法对 调查数据进行整理、分析。

因此, 现状调查应重点注意: 首先要用数据说话; 其次是
整理数据、有效分类、有效分层分析, 直到发现问题症结所 在; 再次, 应到现场观察、跟踪、测量, 把握问题的实质。

(4)现状调查可用工具: 简易图表、排列图、散布图、直方 图、折线图、控制图、分层法、调查表等。

\section{3 设定目标方面}

(1) 该环节工作在于: 确定解决问题到什么样程度, 为活 动的效果检验提供参照依据。

(2)常见失误: a. 选题之后, 而没有设定目标或所设目标 没有数量化。b. 先设定了目标再进行现状调查。c. 目标设定过 多。d.目标设定的依据不充分。

(3)失误分析与应对: 选题之后, 没有设定目标或所设目 标没有数量化, 直接造成无法检查活动是否实现预期效果和 课题目标; 除指令性目标程序外, 其他都应在现况调查之后 定设目标, 只有这样的目标才有依据, 事先设定目标而后做 调查, 是不符合活动准则的程序要求的; 设定多个目标, 会分 散人员精力、复杂化问题、增加实现目标的难度, 因此, 问题 解决型课题一般只设一个活动目标; 目标设定的理由不充 分, 想要目标实现也很难, 如把产品合格率从 $60 \%$ 提高至 99\%，这种大幅度的提高必须具体说明这样设定目标的理 由,应从数据分析、测算过程等来说明, 使其更有针对性、说 服力。

因此,设定目标要做到: 目标同问题要相对应, 且 1 个目 标为宜; 目标要明确表示; 要说明制定目标的理由。

另外, 对指令性的课题, 不需要经过事先“现状调查”, 但 可以直接分析“目标的可行性”,分析应该指出“现实”与“目 标”之间的差别距离多少, 出现差别距离的症结在哪里。

(4)设定目标运用的工具: 柱状图、折线图等。

\section{4 原因分析方面}

(1)这一环节工作在于:工作之前, 把现实状与目标值之间 的差别距离调查分析清楚;工作之时, 充分展开思路, 想到“可 想到的所有方面”,并由此集纳“能够产生问题”的全部原因。

(2)常见失误: a. 仅仅分析课题, 没涉及问题症结。b.分析 不彻底全面。c 选用分析工具不当。d.绘制图表不规范。

(3)失误分析与应对: 只对课题进行分析, 或找到主要质 量问题以后还用课题进行分析, 都会让课题与原因分析失掉 联系,因为“原因分析”应该是围绕调查得出的“问题症结”来 进行; 只分析了个别问题, 没有将主要问题逐一进行分析, 原 本应按“5M1E”方法进行原因分析, 实际上却没有做到, 导致 原因分析不全面;原因分析不彻底,导致后环节无法对症下 药,如只指出人素质低下、工艺过程不合理、半成品及材料不 
合格、制度不健全、管理不到位等浅层次的原因,还需要继续 挖掘出深层次的具体原因; 因果关系倒置或将根本没有因果 关系的因素串联在一起进行分析,都是不对的; 因果图、系统 图只适合用于单一问题，问题较多时应分门别类进行分析， 绝不能将几个问题用 1 张因果图或系统图进行分析; 有 2 个 以上问题或有较多相同因素时,要绘制关联图; 因素之间无 关联,无需绘制关联图,否则会导致关联图变成系统图; 关联 图中问题与因素绘制的图框没有区分或不合规定,系统图的 连接线是直线,因果图、关联图的因素之间是用箭线连接,都 要符合要求; 将方法类中的原因归到人员类别中属原因归类 不准确; 箭线的箭头方向标注错误、因果关系表述逻辑倒置、 硬性地将无因果关系的两原因组合成因果关系, 这些都属因 果关系错误; 标注不全的错误主要包括图表下方绘图人、复 核人名称缺少,图号、图名、绘图日期缺少等。

(4)分析原因应注意:一是要正对 “存在的问题症结”进行 分析原因; 二是分析原因要展示问题的全貌即做到“全面”; 三是分析原因要做到“彻底”即分析要透底;四是要“正确、恰 当”地应用统计方法 (工具), 同时要从“5MIE”的 6 种角度展 开分析。

(5)分析原因的工具有:因果图、系统图、关联图。

\section{5 确定主要原因方面}

(1)这一环节工作在于: 对诸多原因进行鉴别, 把导致问 题的主要原因找出来。

(2)常见失误: a. 没有客观分析就主观地直接确定了原 因, 或者要因确认过于简单, 分析不彻底, 达不到要求, 导致 确认要因依据不足。b.未能逐一确认全部末端原因; 确认要 因时, 只确认其中一部分, 舍弃了其余部分, 造成要因确认错 误。c. 确认要因的方法不得当。d.判定要因时, 既不按原因对 应的问题来判定, 也不按目标实现的影响程度来判定, 而是 根据其是否“容易解决”或是否 “符合标准” 要求来判定。

(3)失误分析与应对: 态度主观、要因确认未用科学方法 工具、分析不全面、彻底, 是造成这类问题的根源。“主要原 因”的确定, 首先是要从在上一步骤中找出的“全部末端原因 之中” 去挖掘, 逐一加以辨别确认; 其次确认的方法是“现场 验证”, 如到现场实地测量、查阅记录、试验等, 而不能主观确 定; 再次是判断的原则, 严禁用分析论证法、举手表决、“是 否容易解决”等为原则确定主要原因。

(4)确定主要原因步骤是: 收取所有末端原因,并剔除末 端因素中不可抗拒的因素对末端原因逐条确认; 通过现场测
量、试验及调查分析, 依据末端原因对问(症结) 影响程度找 出主要原因。

(5)确定主要原因常用工具: 调查表、简易图表、散布图、 直方图。

\section{6 制定对策方面}

(1)这一环节工作在于: 对确定的每一条主要原因制定相 应对策。

(2)常见失误: a. 对策和措施不够完善; b. 对策表中制定的 目标存在缺陷; $c$. 对策表中的责任分工不明确; $d$. 对策表中时 间不准确; e. 语言表述抽象。

(3)失误分析与应对:对策与措施“两者并一”“者缺一” 和“对策表中的措施没有列出具体步骤”, 造成对策与措施不 完善, 实践中的“对策” 是针对主要原因提出的“做什么”, 相 应“措施”应强调“怎么做”。“目标”栏目缺项, 只有定性目标, 无定量目标, 导致实施完成后, 无法检验对策是否完成且达 标; 由课题目标分解而直接形成对策目标, 是不对的, 对策目 标必须是针对主要原因采取措施后所达到的目标。落实责任 分工, 应尽力地把对策措施的实施有效地落实到小组每位成 员身上,让每个人都能发挥作用。对策表中完成时间不具体 或模糊是不符合要求的, 如“本季度” “下半年” 等, 明确具体 的年月日, 便于对照检查和 QC 组长督促节点时间。使用抽 象的词语进行表述, 如加强、提高、争取、尽量、随时等词都非 常含糊、不明确, 不符合以实为主的 QC 小组活动精神, 也不 利于具体工作的指导实施。

(4)制定对策有以下步骤：根据主要原因逐条提出对策; 研究、确定所采取的对策; 制定对策表(按“5WlH”原则)。

(5)制定对策一般用的工具有: 简易图表、矩阵图、矢线 图、优选法、正交试验设计法等

\section{4 结语}

$\mathrm{QC}$ 小组活动是一个完整的 PDCA 过程, 除 $\mathrm{P}$ 过程外的 其他工作环节也很重要。熟练地运用 QC 小组进行质量管 理, 提高企业的质量效益, 必须提升活动各环节的精准定位 水平。

\section{参考文献}

[1] T/CAQ 10201-2016 质量管理小组活动准则[S].

[2] 刘小燕浅谈 QC 小活动中应注意的几个关键环节[J].北京: 科技 与生活, 2010(13):205.

[3] 马东升, 郭立清, 解玉琳.消除问题解决型课题 QC 活动质量通病 的探讨[J].北京:市政技术,2018,36(5):242-246. 